\title{
Substances psychoactives et travail. Approche épidémiologique
}

\author{
Psychoactives drugs and workplace. \\ An epidemiological approach
}

\section{Bernard FONTAINE*(1), Groupe Régional Toxicomanies et Travail $^{(2)}$}

(1) Comité pour le Développement de la Médecine du Travail, 118, rue Solférino - 59000 LILLE

(2) Institut Santé Travail du Nord de la France, 1, avenue Oscar Lambret - 59000 LILLE

* Auteur à qui adresser la correspondance : Bernard FONTAINE, 118, rue Solférino - 59000 LILLE Tél : 0320128300 - Fax : 0320782589

(Reçu le $1^{\text {er }}$ février 2002 ; accepté le 20 février 2002)

\section{RÉSUMÉ}

La connaissance épidémiologique des toxicomanies en milieu professionnel est freinée par des obstacles d'ordres divers.

- Éthique : information préalable et accord quant à la nature de la recherche.

- Technique : les médecins du travail ont besoin d'une réponse immédiate, car laisser une aptitude en attente peut nuire au salarié.

- Financier : le coût (en particulier la confirmation en $C G / S M)$ représente un obstacle pour certaines entreprises.

- Temporels : le temps consacré aux examens médicaux ne peut augmenter.

- Réglementaire : une politique de dépistage systématique et organisé chez les salariés affectés à un poste de sûreté/sécurité (PSS) doit être prévue au règlement intérieur.

- Culturel : nécessité de placer l'alcool au même rang que les autres psychoactifs.

- Pratique : le rapport facilité d'utilisation/sensibilité/spécificitéladéquation aux besoins du monde du travail des tests

\section{SUMMARY}

Medical examination of the workforce is not sufficient by itselffor assessing the problem. So it's necessary to complete it by a biological testing, which is difficult for many reasons, belonging to different ranks.

- Ethics : workers must have been warned of the nature of the biological testing, and in agreement with it.

- Technics : occupational physicians need quick on site results to be able to allow the fitness of the workers to the proposed job.

- Money : the analysis coast (especially GC/MS) is unaffordable for some enterprises.

- Timing : impossibility of increasing unceasingly duration of medical examinations.

- Regulation : systematic drug testing is impossible, even in the safety security workforce, except in case of specific internal rules allowing it.

- Convenience : the different instant tests now on sale are not yet sufficiently fitted for an easy use in terms of sensibility, specificity, fitness to the proposed goal. 
instantanés disponibles est insuffisamment performant. Une étude portant sur les urines anonymisées de 1976 salariés du Nord Pas-de-Calais quasi représentatifs de la population salariée régionale, a été réalisée en 1995 en vue d'appréhender par recherche immunoenzymatique la consommation de cannabis, opiacés, amphétamines, cocaïne, propoxyphène, alcool, benzodiazépines et barbituriques. Les médecins préleveurs n'ayant pas le résultat des urines confiées au laboratoire, l'éthique était respectée car aucune conséquence n'était à craindre sur l'aptitude des salariés enquêtés à leur insu : le conseil de l'ordre des médecins avait d'ailleurs donné son accord préalable.

Le pourcentage de salariés consommant au moins une substance psychoactive était de 17,6\%, mais au niveau des PSS atteignait $40 \%$ !

Depuis, d'autres travaux ont été réalisés en particulier dans le monde du transport routier, et dans diverses entreprises, travaux dont une tentative de synthèse sera tentée.

\section{MOTS-CLÉS}

Toxicomanies, lieux de travail, épidémiologie.

\section{Introduction}

Le monde du travail n'échappe pas à l'augmentation constatée partout de la consommation de substances psychoactives. Les difficultés d'appréhension du phénomène sont nombreuses, rendant difficile une approche épidémiologique rigoureuse, mais les nécessités de la prévention imposent une connaissance "raisonnable" du phénomène à l'échelon global pour pouvoir établir une stratégie à la fois éthique et pertinente. En effet, seules des enquêtes de prévalence réalisées avec l'aide de médecins du travail peuvent apporter aux décideurs les arguments indispensables à la prise de mesures proportionnées à l'ampleur des conséquences potentielles sur la sécurité dans les entreprises. Il va être détaillé dans cet article une étude réalisée en 1995 dans la région Nord Pas-de-Calais (1), dont les insuffisances seront analysées, mais qui reste cependant à notre connaissance le seul travail de ce type et de cette ampleur dans le monde du travail interprofessionnel. Nous étudierons ensuite les obstacles à la réalisation de nouvelles études, en tentant d'y apporter des solutions.

\section{Matériel et méthodes}

La conformité à l'éthique du protocole d'enquête est un préalable indispensable à ce type de travail. C'est pourquoi l'information et l'accord préalable du Comité Régional d'Ethique et du Conseil Départemental de l'Ordre des Médecins ont été obtenus avant le début de celle-ci. Ce protocole prévoyait le recueil systématique des urines de tous les salariés vus en visite d'embauche ou en visite périodique un lundi matin ou un jeudi matin, sans que les salariés ne soient avertis des
So as to bypass these difficulties a study of 1976 urines from 1976 workers of the Nord Pas-de-Calais area, who were quite representative of the regional workforce, was performed in 1995 in order to assess, by immunoenzymatic assay, the use of cannabis, opiates, amphetamines, cocaine, propoxyphene, alcohol, benzodiazepines and barbiturates. It was in respect with ethics, while none individual result was addressed to the physicians, and so, none adverse outcome was possible towards the workers who had not been warned before the test: the local medical order council was in agreement with that study.

17,6\% of the workforce used at least one psychoactive drug, but, for the safety security workforce, the percentage reached $40 \%$.

Since that study, other ones were conducted, especially among truckdrivers, and we will try to resume them.

\section{KEY-WORDS \\ Drug addiction, workplace, epidemiology.}

dosages qui allaient être pratiqués : il était donc indispensable que les médecins préleveurs ne puissent donc, a posteriori, modifier leur décision d'aptitude en connaissant nominativement les résultats de leurs salariés. C'est la raison pour laquelle ne figuraient sur les flacons que les renseignements suivants : sexe, âge, lundi ou jeudi, origine géographique sommaire du prélèvement, affectation ou non à un poste de sûreté sécurité (PSS). Ce dernier critère était laissé à l'appréciation des médecins du travail enquêteurs.

Les recherches ont porté sur les 8 produits ou classes de produits suivants : cannabis, opiacés, amphétamines, cocaïne, barbituriques, benzodiazépines, propoxyphène et éthanol, et ont été effectuées en immunoenzymatique par la méthode EMIT.

Au total, 1978 urines de salariés de la région Nord Pasde-Calais ont été recueillies, provenant de 13 services différents de médecine du travail volontaires pour participer à l'enquête qui s'est déroulée en janvier-février 1995.

\section{Résultats}

La structure démographique de la population testée est la suivante : $72 \%$ d'hommes, d'âge moyen 37,6 ans \pm 10,7. Une sur représentation masculine est constatée (en 1990, la population active du Nord Pas-de-Calais (données INSEE) comptait $58 \%$ d'hommes), tandis que la classe d'âge 56-65 ans (4\%), quoique conforme à celle suivie en médecine du travail à l'époque, était plus faible que les 9,2\% annoncés par l'INSEE. La figure $\mathrm{n}^{\circ} 1$ présente les classes d'âge de la population enquêtée. 
Si la répartition géographique des salariés donnait une représentation correcte de la population du Nord, il n'en était pas de même dans le Pas-de-Calais où seuls les services de médecine du travail de l'ex bassin minier, participaient à l'étude : il en ressort donc également un déséquilibre numérique entre le Nord $(88,3 \%)$ et le Pas-de-Calais $(11,7 \%)$ au niveau du nombre d'urines collectées. Enfin, un quart des salariés était affecté à un PSS.

La prévalence globale des consommations de psychoactifs est présentée dans le tableau I. On voit donc que près d'un salarié sur 4 (23,3\% chez les hommes, $24,1 \%$ chez les femmes) consomme au moins une substance pouvant affecter sa vigilance et/ou son comportement au travail. Les hommes consomment plus de cannabis et d'alcool que les femmes qui elles, préfèrent les benzodiazépines, amphétamines et propoxyphène. Aucune consommation de cocaïne n'a été décelée.

La figure 2 renseigne sur le profil de consommation par produits en fonction de l'âge. Les jeunes préfèrent le cannabis et, de façon moins nette, les amphétamines, tandis que les autres produits sont préférentiellement consommés par les classes d'âge plus âgées.

Sans que la différence soit flagrante, la consommation de week-end d'alcool et de cannabis est mieux vue le lundi que le jeudi.

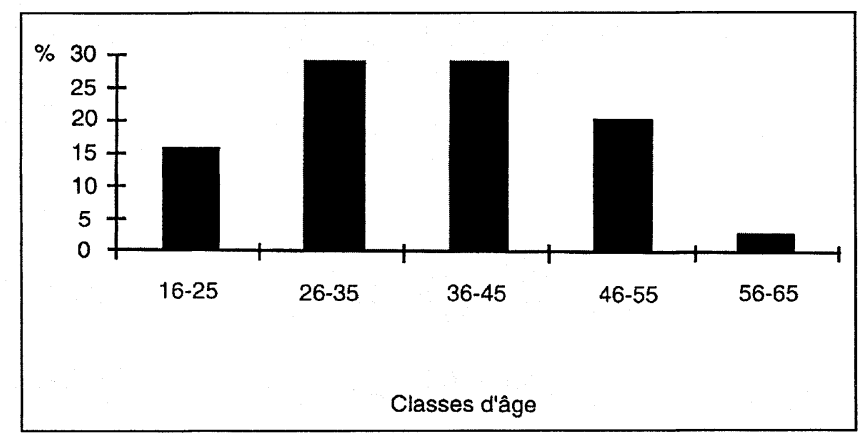

Figure 1 : Caractéristiques démographiques de la population testée.

Tableau I : Prévalence des différentes substances recherchées sur la population active testée (méthode EMIT).

\begin{tabular}{|l|c|c|c|}
\hline Paramètres & \% positifs & $\%$ de femmes & \% d'hommes \\
\hline Opiacés & 5,62 & 5,00 & 5,80 \\
\hline Cannabis & 4,81 & 3,80 & 5,10 \\
\hline Benzodiazépines & 4,07 & 6,30 & 3,30 \\
\hline Éthanol & 3,00 & 0,97 & 3,80 \\
\hline Amphétamines & 2,90 & 4,30 & 2,50 \\
\hline Propoxyphène & 1,52 & 2,30 & 1,30 \\
\hline Barbituriques & 1,46 & 1,40 & 1,50 \\
\hline Cocaïne & 0 & 0 & 0 \\
\hline
\end{tabular}

Sur le plan géographique, le profil des consommations est également différent, le bassin minier consommant plus d'opiacés et le dunkerquois plus de cannabis.

Plus intéressante et novatrice est l'étude de l'influence de l'affectation à un PSS (poste de sécurité-sureté) sur la prévalence de consommation, montrée sur le tableau II.

Bien qu'il n'existe pas de définition légale des PSS, il existe un consensus assez fort parmi les médecins du travail sur la liste indicative suivante, établie par les membres du groupe régional Toxicomanies et Travail, liste indicative et non limitative des postes de sûreté sécurité pour lesquels l'usage de substances psychoactives est interdit au même titre que l'alcool : Risque de brûlure/explosion, risque de noyade, risque biologique ou chimique, risque nucléaire, mines et carrières, travail en hauteur, travail isolé ou en horaires décalés, travail sur machines dangereuses, conduite d'engins de chantier, de manutention, de levage, conduite professionnelle (y compris VL), métiers de contrôle de la sécurité collective, accès par badge à une zone sensible sans contrôle, secteur sanitaire et social, postes clés informatiques, postes de management ou décisionnel, services financiers, pilotage de process, accès à des toxiques industriels ou à des médicaments, port d'arme, service de gardiennage et d'incendie....

On voit donc que 4 salariés sur 10 affectés à un PSS sont consommateurs, et que, à part les barbituriques et les benzodiazépines, toutes les autres classes de produits sont nettement concernées.

\section{Discussion}

Le premier point faible de cette étude est, bien évidemment, la non confirmation en chromatographie en phase gazeuse couplée à la spectrométrie de masse, qui en particulier pour les amphétamines et les opiacés est indispensable. Cette confirmation était initialement prévue, mais n'a pu se faire pour des raisons budgétaires. On peut cependant supposer que la nette sur prévalence de consommation d'opiacés par les salariés les plus âgés est en rapport avec la pathologie bronchique hivernale, l'enquête ayant eu lieu en saison froide. A l'inverse, dans une optique pratique, peu importera au médecin du travail que le produit consommé soit légal ou illégal, médicamenteux prescrit ou médicamenteux détourné, seule la répercussion de cette consommation sur l'aptitude professionnelle sera décisive.

Le deuxième point faible est son caractère régional, le Nord Pas-de-Calais étant bien connu comme une région à haut risque de toxicomanies.

Les autres faiblesses de ce travail sont, en dehors de son ancienneté (7 ans actuellement), et de la non cou- 


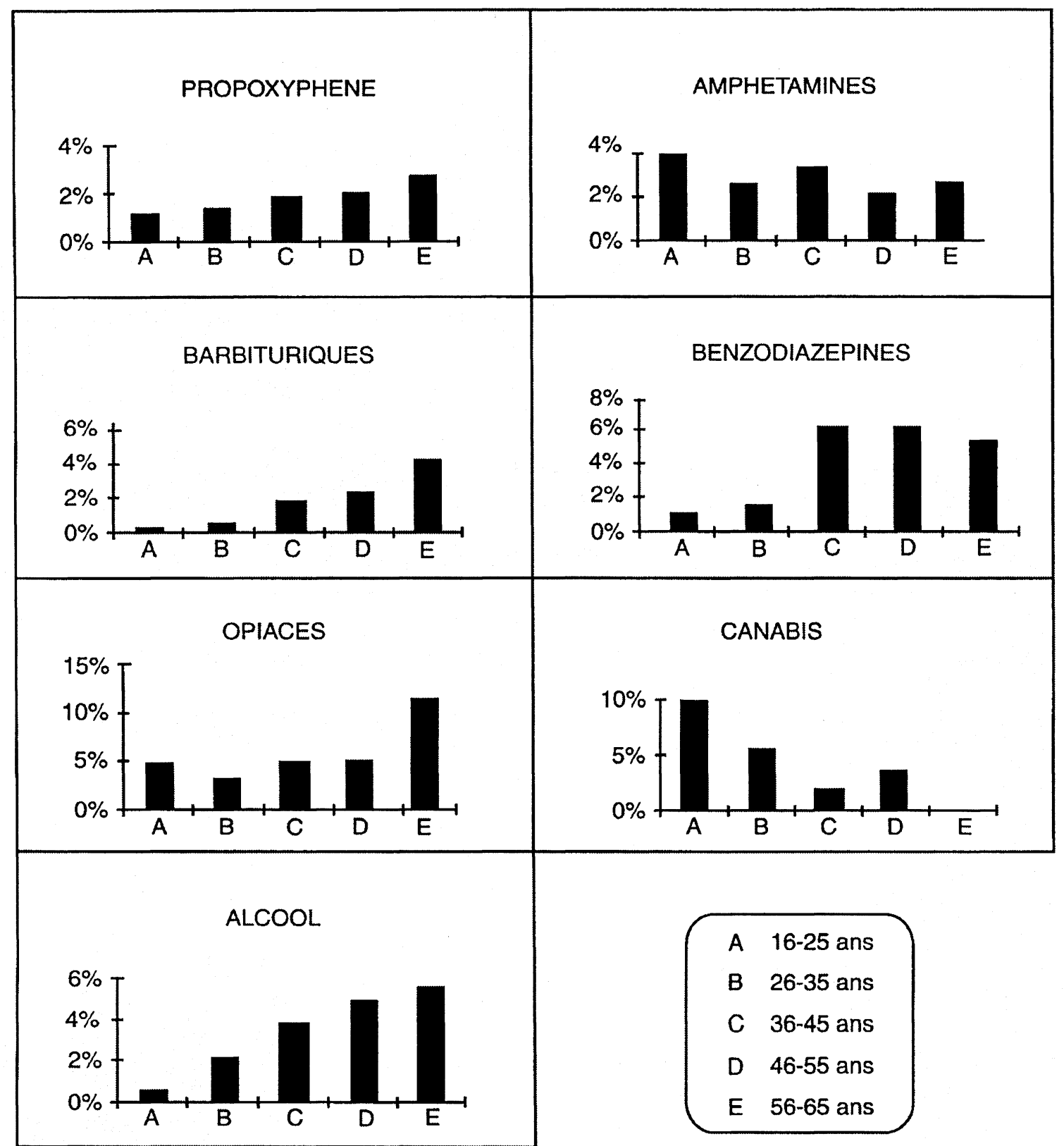

Figure 2 : Prévalence des différentes subtances testées selon les classes d'âge (Méthode EMIT).

Tableau II : Prévalence des différentes substances testées selon l'affectation ou non à un poste de sécurité (PSS) (méthode EMIT).

\begin{tabular}{|l|c|c|}
\hline PSS & + & - \\
\hline Paramètres & $\%$ & $\%$ \\
\hline Cannabis & 10,20 & 4,50 \\
Opiacés & 12 & 5,40 \\
Propoxyphène & 2,40 & 1,70 \\
Amphétamines & 5,20 & 3,20 \\
Éthanol & 5,40 & 1,50 \\
\hline Benzodiazépines & 3,80 & 4,30 \\
Barbituriques & 1,40 & 1,50 \\
\hline
\end{tabular}

verture de certains domaines professionnels (fonction publique, agriculture, armée, secteur artisanal,...), l'appréhension d'une consommation par un dosage urinaire, et non par un dosage sanguin mieux corrélable à une modification du comportement ou du niveau de vigilance. Enfin, l'explosion de la prescription de buprénorphine depuis la réalisation de cette étude est un point qui devient capital à appréhender dans le monde du travail, tout comme la consommation de cocaïne et dérivés dont nous savons qu'elle existe maintenant chez des salariés de notre région.

A l'inverse, et malgré ces faiblesses, posséder une première approche de prévalence de consommation, avoir 
traité la consommation de psychoactifs comme celle d'éthanol et avoir établi l'existence d'une nette sur consommation chez les salariés affectés à des PSS incite à aller plus loin dans la recherche.

Depuis cette enquête, un travail portant sur une population de 313 postulants chauffeurs routiers (2) a été récemment présenté : entre l'entretien préalable à l'embauche, où les chauffeurs sont avertis de la réalisation d'un test, et la réalisation de la visite d'embauche par le médecin du travail un mois plus tard, il y a $10 \%$ de désistement spontané. Dans la population présente effectivement à l'examen d'embauche $(95 \%$ de la population de moins de 35 ans), on retrouve $11 \%$ de consommateurs de cannabis et $2 \%$ de consommateurs d'opiacés.

\section{Conclusion}

Une enquête nationale de prévalence dans le monde du travail est maintenant nécessaire. En attendant la réalisation de celle-ci, des enquêtes par branches professionnelles doivent être initiées rapidement, en particulier dans les professions où la conduite de véhicule représente une partie ou la totalité de la tache à accomplir. La promulgation récente du décret $\mathrm{n}^{\circ} 2001-751 \mathrm{du}$ 27 août 2001 et de la loi $n^{\circ} 2001-1062$ du 15 novembre 2001 devraient être des starters dans le déclenchement de nouveaux travaux, et aider à lever certains obstacles culturels et réglementaires (égalité avec l'alcool). Sur le plan éthique, l'inclusion au règlement intérieur d'une liste consensuellement établie au sein des entreprises et l'adoption clairement annoncée d'une politique de dépistage à l'embauche et de façon périodique et aléatoire chez les salariés affectés à l'un des postes de cette liste permettent de concilier à la fois la protection de l'être humain dans sa singularité et dans son genre.

Les aspects techniques et pratiques sont, par rapport à 1995, nettement simplifiés par la qualité des tests instantanés proposés actuellement (3), tests qui doivent évoluer pour mieux s'adapter à la spécificité du monde du travail, mais qui ne pourront jamais cependant égaler la CG/SM qui doit rester l'examen de confirmation quand celle-ci est nécessaire.

\section{Références}

1. Haguenoer J.M., Hannothiaux M.H., Lahaye-Roussel M.C., Fontaine B., Legrand P.M., Shirali P., Pamart B., Brillet J.M., Brouck N., Bailly I., Frimat P. Prévalence des comportements toxicophiles en milieu professionnel : une étude dans la région Nord Pas-de-Calais. Bull. Conseil Départ. du Nord Ordre Méd., 1997, 80, 11-5.

2. Delzenne C., Pradeau P. Limites, intérêt et avenir du dépistage systématique des substances psychoactives, étude sur une population de chauffeurs routiers. $\mathrm{XXI}^{\mathrm{emes}}$ Journées Méditerranéennes Internationales de Médecine du Travail. 26-28 oct. 2001, Marrakech.

3. Fontaine B., Legrand P.M., Pamart B. Eloy E., Frimat P., Vignau J., Lhermitte M. Évaluation de 7 tests rapides pour le dépistage des substances psychoactives dans le cadre de la médecine du travail. Documents pour le médecin du travail, $\mathrm{n}^{\circ} 87,3^{\text {eme }}$ trim. 2001, 321-4. 